\title{
CONTROLE DA MATURAÇÃO DE CAQUIS 'FUYU', COM USO DE AMINOETHOXIVINILGLICINA E ÁCIDO GIBERÉLICO ${ }^{1}$
}

\author{
VALDECIR CARLOS FERRI ${ }^{2}$, MARIA MADALENA RINALDI ${ }^{3}$, ROQUE DANIELI ${ }^{3}$, \\ LUCIANO LUCHETTA ${ }^{4}$, CESAR VALMOR ROMBALDI ${ }^{5}$
}

\begin{abstract}
RESUMO - Avaliaram-se o efeito do controle da maturação e o comportamento pós-colheita de caquis 'Fuyu', tratados a campo com aminoethoxivinilglicina ( $\mathrm{AVG}$ ) e ácido giberélico $\left(\mathrm{AG}_{3}\right)$. Utilizou-se o delineamento completamente casualizado, com quatro repetições. As pulverizações foram realizadas com $\mathrm{AVG}$ a 50ppm e $\mathrm{AG}_{3}$ a 30ppm, 30 dias antes da data prevista para a colheita. Após a colheita, os frutos foram armazenados em ambiente com temperatura $23 \pm 3^{\circ} \mathrm{C}$ e umidade relativa de $75 \pm 5 \% \mathrm{e}$, a cada quatro dias, foram realizadas avaliações da perda de peso, firmeza de polpa, produção de etileno e teor de clorofilas e de carotenóides. As aplicações de $\mathrm{AG}_{3}$ e de AVG permitiram retardar o momento da colheita dos frutos e auxiliaram no armazenamento dos mesmos, através da preservação da integridade física dos frutos.
\end{abstract}

Termos para indexação: regulador de crescimento, conservação, Diospyrus kaki,

\section{MATURATION CONTROL OF KAKI 'FUYU' USING AMINOETHOXIVINILGLICIN ADN GIBBERELIC ACID}

\begin{abstract}
This paper aims to evaluating the effect of maturation control and the post-harvest behavior in relation to Kaki 'Fuyu' sprayed with aminoethoxivinilglicin (AVG) and Gibberelic acid $\left(\mathrm{AG}_{3}\right)$ in the field. As to spraying, $\mathrm{AVG}$ at $50 \mathrm{ppm}$ and $\mathrm{AG}_{3}$ at $30 \mathrm{ppm}$ were used 30 days before harvest with four repeated applications at random. The fruits were stored at average temperatures of $23 \pm 3^{\circ} \mathrm{C}$ and relative humidity of $75 \pm 5 \%$; weight loss, pulp firmness, ethylene production, chlorophyll and caretenoid substances were evaluated every four days. The $\mathrm{AG}_{3}$ and $\mathrm{AVG}$ applications permited to retard harvest and helped storing of the fruits, through the physical structure preservation of fruits.
\end{abstract}

Index terms: growth regulator, preservation, Diospyrus kaki.

\section{INTRODUÇÃO}

O caquizeiro (Diospyrus kaki, L.) é uma espécie originária da Ásia, onde é cultivada há mais de cinco séculos. Entretanto, é no Japão que este fruto tem maior importância.

A cultivar Fuyu é a principal representante da boa adaptação de seu cultivo nas Regiões Sul e Sudeste do Brasil. Seu período de colheita é de aproximadamente 30 dias, quando mais de $90 \%$ da produção é comercializada imediatamente após a colheita.

Para ampliar o período de colheita e o armazenamento deste fruto, necessita-se estabelecer novas condições de manejo na pré-colheita, na colheita e no armazenamento, tais como, o emprego de reguladores de crescimento e/ou de reguladores de vias metabólicas da maturação, permitem além do controle parcial da maturação e senescência, e reduzir os distúrbios fisiológicos nos frutos (Kende, 1993; Pechet al., 1994; Looney, 1998).

De acordo com alguns autores (Byers, 1997; Petri \& Leite, 1999), a aplicação de aminoethoxivinilglicina (AVG), além de controlar a maturação, age no processo de floração, inibindo o aborto de flores, e estimula o crescimento vegetativo em caquizeiros.

Aplicações do AVG inibiram a síntese de etileno durante o armazenamento de maçãs (Dennis et al., 1983), pêssegos (Byers, 1997) e caquis (Ben-Arie \& Zutkhi, 1992). Entretanto, em caquis, sua eficiência depende da cultivar, em especial da produção e de sua sensibilidade ao etileno (Autio \& Bramlage, 1982).

O uso do ácido giberélico $\left(\mathrm{AG}_{3}\right)$ em pulverizações précolheita, durante as fases de crescimento e de expansão celular de tangerinas (Barros \& Rodrigues, 1994), também tem sido recomendado como forma de controle da maturação (retardando o período de colheita), de manutenção da qualidade pós-colheita (aumento da conservação) e de retardo no processo de senescência, fato que também tem sido observado para caquis (Kang et al., 1994; Perez et al., 1995; Ben-Arie et al.,1996) o que possibilita escalonar a colheita e prolongar sua oferta.

Em se tratando de uma cultura ainda em início de exploração nos Estados do Rio Grande do Sul e Santa Catarina, procurou-se estudar o efeito da $\mathrm{AVG}$ e do $\mathrm{AG}_{3}$, no controle da maturação e no comportamento pós-colheita de caquis, cultivar Fuyu, armazenados em condições ambientais.

1 (Trabalho 073/2001). Recebido: 27/03/2001. Aceito para publicação: 02/05/2002. Artigo que faz parte da Tese de Doutorado do primeiro autor.

2 Engo Agro , Doutor em Ciências Agrárias, Professor de Viticultura do CSTVE da EAFPJK - Bento Gonçalves, RS.

3 Eng $^{\mathrm{o}} \mathrm{Agr}^{\mathrm{o}}$, Mestre em Ciência e Tecnologia Agroindustrial

4 Bolsista CNPq, acadêmico em Agronomia na UFPel/FAEM.

5 Eng ${ }^{\mathrm{o}}$ Agro $^{\circ}$, Dr. Prof. Adjunto do Departamento de Ciência e Tecnologia Agroindustrial da UFPel/FAEM.

Rev. Bras. Frutic., Jaboticabal - SP, v. 24, n. 2, p. 344-347, agosto 2002 


\section{MATERIAL E MÉTODOS}

O experimento foi conduzido em pomar comercial da cultivar Fuyu, localizado no município de Canguçu - RS, na safra 1998/99, o qual é formado por plantas com 12 anos de idade, e espaçamento de 2-3 metros.

O delineamento experimental adotado seguiu uma orientação completamente casualizada, com quatro repetições, seguindo esquema fatorial $3 \times 5$ (tratamentos a campo $\mathrm{x}$ período de armazenamento).

Os caquizeiros foram tratados a campo (21 de fevereiro), 30 dias antes da data prevista para a colheita dos frutos, através de pulverizações totais até gotejamento, que foram: 1 caquizeiros pulverizados com aminoethoxivinilglicina (AVG), na dosagem de $125 \mathrm{~g} \mathrm{ia} \mathrm{ha}^{-1}$ (a fonte de AVG foi o produto comercial RetainÒ, a 50ppm em água, contendo $0,02 \% \mathrm{v} / \mathrm{v}$ de espalhante aniônico SilvetÒ; 2 - pulverização das plantas com ácido giberélico $\left(\mathrm{AG}_{3}\right.$ ), na dosagem de $75 \mathrm{~g}$ ia ha ${ }^{-1}$ (a fonte de $\mathrm{AG}_{3}$ foi o produto comercial Progibb $^{\circledR}$, a 30ppm em água); e 3 - pulverização com água (controle).

O ponto de colheita dos caquis foi estabelecido através da firmeza da polpa $(70 \pm 5 \mathrm{~N})$ e da coloração da epiderme (verdeamarelada).

A partir da colheita, os caquis foram mantidos em ambiente com $23 \pm 3^{\circ} \mathrm{C}$ e $75 \pm 5 \%$ de umidade relativa, e a cada quatro dias, durante 20 dias, foram submetidas às análises de: perda de massa fresca, determinada pela diferença entre a massa inicial e aquela no momento da realização das análises, expressa em porcentagem (\%); firmeza de polpa, expressa em Newton (N), determinada por penetrômetro manual, munido de ponteira de oito milímetros de diâmetro, com determinações em faces opostas, na região equatorial dos frutos, após a remoção da casca; produção de etileno, determinada por cromatografia em fase gasosa, cujas temperaturas da câmara de injeção, coluna e detector foram de $80^{\circ} \mathrm{C}, 90^{\circ} \mathrm{C} \mathrm{e} 200^{\circ} \mathrm{C}$, respectivamente. Utilizou-se, como padrão, uma solução de etileno a 10ppm. Para a determinação da produção de etileno, dois frutos eram acondicionadas em frascos hermeticamente fechados, durante uma hora, a $25^{\circ} \mathrm{C}$. Ao vedar-se os frascos e passado o período de acondicionamento, era coletado, com auxílio de seringas hipodérmicas, $1 \mathrm{ml}$ da atmosfera gasosa. A quantificação foi feita, correlacionando-se a média das alturas dos picos relativos a cada amostra, com a média das alturas dos picos obtidos da solução-padrão de etileno, a qual foi expressa em $\mathrm{nL} \mathrm{g}^{-1} \mathrm{~h}^{-1}$; o teor de clorofilas e de carotenóides, determinados através das metodologias descritas por Hill et al. (1985) e Ramojaro et al. (1979), respectivamente, com resultados expressos em $\mathrm{mg} \mathrm{g}^{-1}$ de peso fresco dos frutos.

As amostras, compostas por 12 frutos, foram padronizadas e acondicionadas em bandejas plásticas individualizadas. Os frutos destinados a avaliação do etileno foram os mesmos desde o início das leituras.

Os resultados foram submetidos à análise de variância, para a comparação de médias dos tratamentos, utilizando-se do teste de Duncan, a 5\% de probabilidade. Para o estudo das variações durante a maturação, adotou-se a regressão polinomial.

\section{RESULTADOS E DISCUSSÃO}

De acordo com os tratamentos, os caquis foram colhi- dos em 23-03 (controle), 04-04 (AVG) e 06-04 ( $\mathrm{AG}_{3}$ ), quando a firmeza de polpa atingiu $75 \pm 5 \mathrm{~N}$ e a coloração da epiderme apresentava-se verde-amarelada.

O emprego da $\mathrm{AVG}$ e do $\mathrm{AG}_{3}$, na pré-colheita, retardou a maturação dos caquis 'Fuyu' em 12 e 14 dias, respectivamente, em relação ao tratamento-controle, com preservação da firmeza da polpa e do conteúdo de clorofila.

Durante o armazenamento, os frutos apresentaram significativa perda de massa fresca, em média $6 \%$, a partir do $12^{\circ}$ dia de avaliação (Figura 1). Isto já era esperado, uma vez que se estabelece um gradiente positivo de pressão de vapor entre os caquis e a atmosfera ambiente $\left(23 \pm 3^{\circ} \mathrm{C}\right.$ e $75 \pm 5 \%$ UR), além da atividade respiratória. As menores perdas foram registradas em frutos tratados com $\mathrm{AVG}$ e com $\mathrm{AG}_{3}$ devido, provavelmente, à menor atividade metabólica.

A firmeza da polpa dos frutos apresentou uma significativa redução em todos os tratamentos durante o armazenamento (Figura 2). Esta redução deve-se à evolução da maturação, que se caracteriza pela ação das enzimas que catalisam o processo de hidrólise dos compostos da parede celular (Kader et al., 1989).

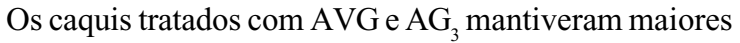
valores de firmeza da polpa em todas as avaliações. Mitchamet al. (1998) indicam a firmeza da polpa de $20 \mathrm{~N}$ como o limite inferior para o consumo de caquis 'Fuyu', pois valores inferiores a este comprometem a estrutura física e o paladar do fruto, indicando que os tratados com $\mathrm{AVG}$ podem ser armazenados por 20 dias. Para os frutos tratados com $\mathrm{AG}_{3}$, este período foi reduzido a 16 dias e, para os não tratados, a 12 dias.

Após a colheita, os frutos apresentavam uma produção de etileno média de $4,9 \mathrm{~nL} \mathrm{~g}^{-1} \mathrm{~h}^{-1}$, indicando que eles foram colhidos já no início da fase climatérica. Durante o armazenamento, chegaram a atingir, no tratamento-controle, até $25 \mathrm{~nL} \mathrm{~g}^{-1} \mathrm{~h}^{-1} \mathrm{de}$ etileno (Figura 3).

Segundo Pech et al. (1994), a produção de etileno permite avaliar a velocidade da maturação. Inicialmente, não houve diferença entre os tratamentos. A partir de quatro dias de estocagem, os caquis começam a diferir, sendo que as menores liberações de etileno foram obtidas nos frutos tratados com $\mathrm{AVGe}_{\mathrm{AG}}$.

Embora o AVG tenha um potente efeito inibidor da síntese do etileno, neste experimento, sua eficiência foi inferior à do $\mathrm{AG}_{3}$. Em outros frutos, a exemplo da maçã, o efeito é contrário (Petri \& Leite, 1999).

Em todos os tratamentos, houve redução no conteúdo de clorofilas. Entretanto, nos tratamentos com $\mathrm{AVG}$ e $\mathrm{AG}_{3}$, preservaram-se os maiores teores de clorofilas nos frutos (Figura 4). No caso dos frutos tratados com $\mathrm{AG}_{3}$, segundo Ben-Arie et al. (1996), estes resultados são conseqüência do efeito do ácido giberélico como inibidor de clorofilases. Já, para o AVG, embora o mecanismo ainda não seja totalmente conhecido, acredita-se que ele também atue inibindo a síntese e/ou a atividade das clorofilases.

Houve influência do $\mathrm{AVG}$ e do $\mathrm{AG}_{3}$ sobre o acúmulo de carotenóides nos frutos (Figura 5). Os frutos que, no momento da colheita, apresentavam níveis de carotenóides médios entre 1,56 e 2,51 $\mathrm{mg} \mathrm{g}^{-1}$, passaram a valores médios entre 3,6 e $5,9 \mathrm{mg} \mathrm{g}$ ${ }^{1}$. O maior acúmulo ocorreu em frutos-controle, e o menor, em frutos tratados com $\mathrm{AG}_{3}$. Na literatura, há referências que citam o efeito protetor do $\mathrm{AG}_{3}$ na degradação de clorofilas (Ben-Arie et al., 1996), mas não o efeito inibidor no acúmulo de carotenóides. 


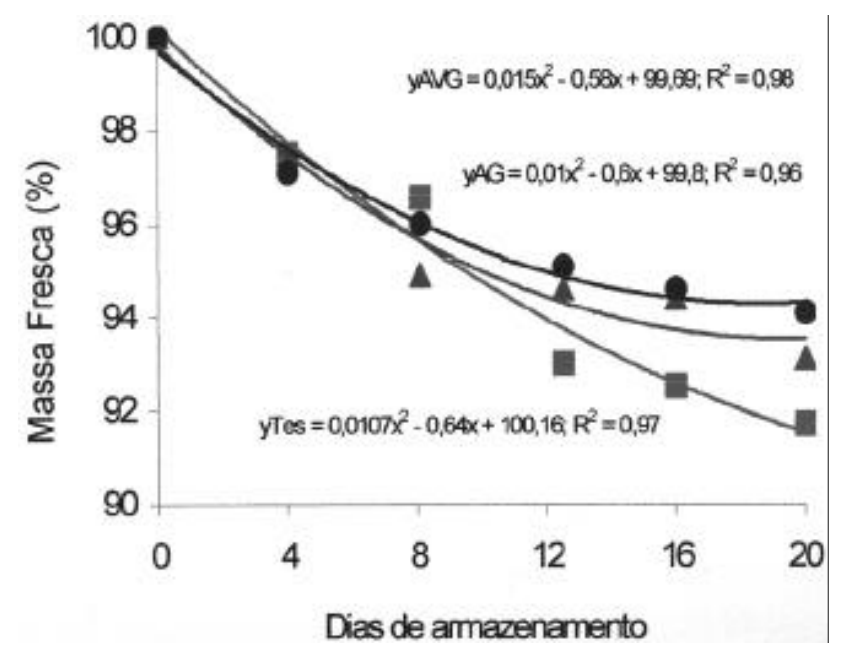

FIGURA 1 - Perda de peso (\%) por caquis 'Fuyu' tratados a campo com aminoethoxivinilglicina (AVG- - - , ácido giberélico ( $\mathrm{AG}_{3}-\mathbf{-}$-) e com água (Controle- - -), e armazenados após a colheita a $23 \pm 3^{\circ} \mathrm{C}$ e $75 \pm 5 \%$ UR.

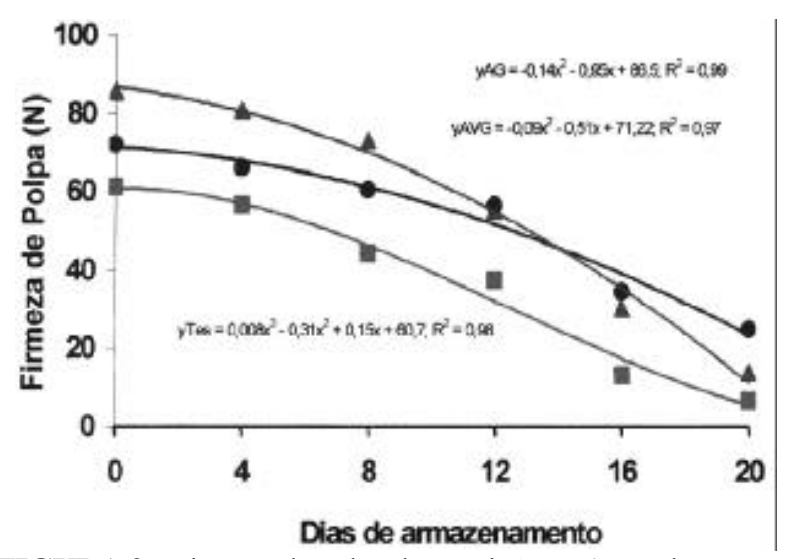

FIGURA 2 - Firmeza da polpa de caquis 'Fuyu' tratados a campo com aminoethoxivinilglicina (AVG- - - , ácido giberélico ( $\mathrm{AG}_{3}-\mathbf{A}$-) e com água (Controle- - -), e armazenados após a colheita a $23 \pm 3^{\circ} \mathrm{C}$ e $75 \pm 5 \%$ UR.

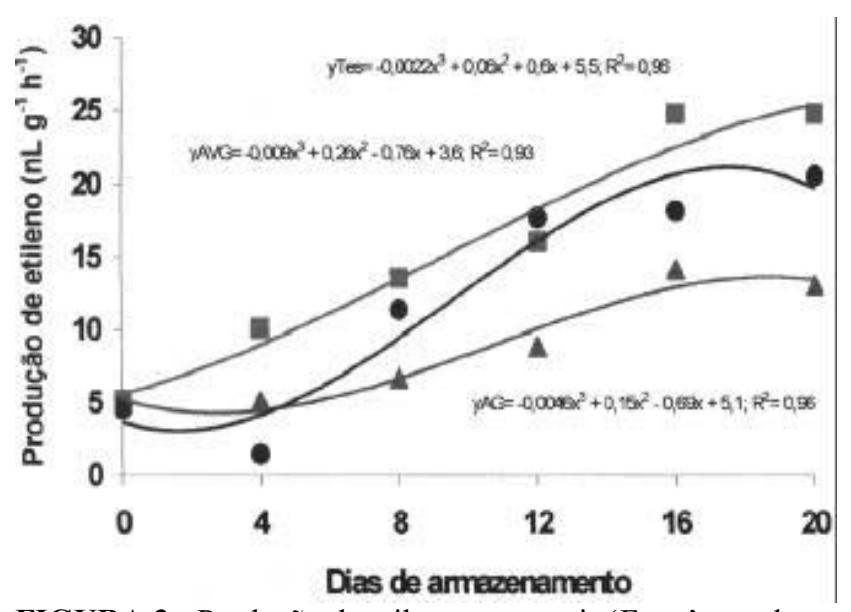

FIGURA 3 - Produção de etileno em caquis 'Fuyu' tratados a campo com aminoethoxivinilglicina (AVG- •-), ácido giberélico $\left(\mathrm{AG}_{3}-\mathbf{\Lambda}\right.$ ) e com água (Controle- - -), e armazenados após a colheita a $23 \pm 3^{\circ} \mathrm{C}$ e $75 \pm 5 \%$ UR.

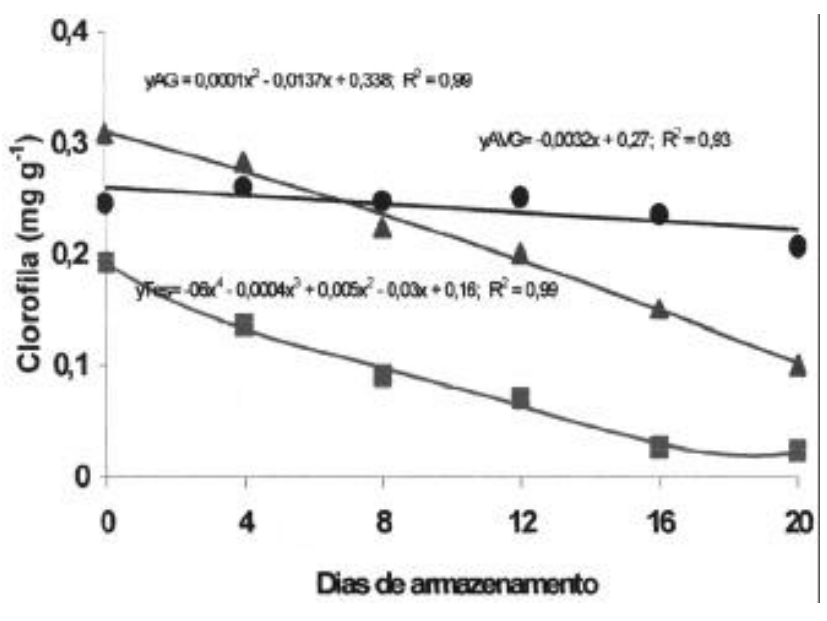

FIGURA 4 - Conteúdo de clorofila em caquis 'Fuyu' tratados a campo com aminoethoxivinilglicina (AVG- - -), ácido giberélico $\left(\mathrm{AG}_{3}-\mathbf{\downarrow}\right)$ e com água (Controle- - ), e armazenados após a colheita a $23 \pm 3^{\circ} \mathrm{C}$ e $75 \pm 5 \% \mathrm{UR}$.

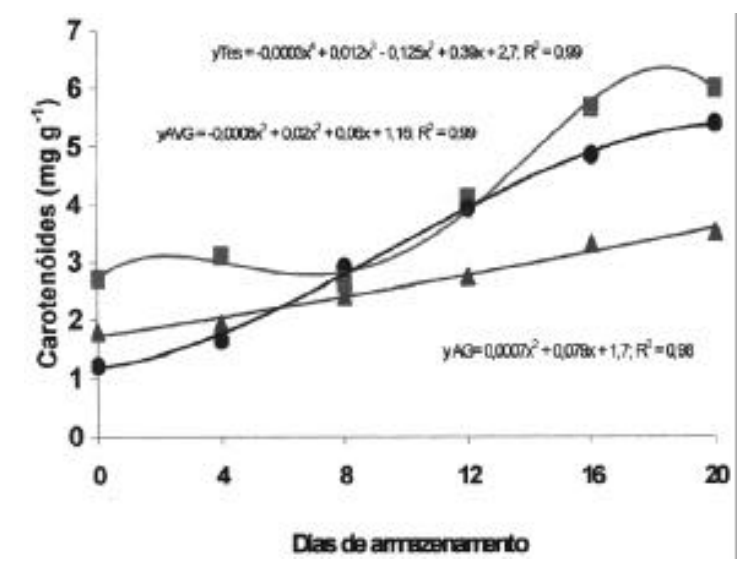

FIGURA 5 - Conteúdos de carotenóides em caquis 'Fuyu' tratados a campo com aminoethoxivinilglicina (AVG- - $)$, ácido giberélico $\left(\mathrm{AG}_{3}-\mathbf{\$}\right)$ e com água (Controle- $\mathbf{-}$-), e armazenados após a colheita a $23 \pm 3^{\circ} \mathrm{C}$ e $75 \pm 5 \%$ UR.

O constante decréscimo no teor de clorofilas (Figura 4) foi acompanhado de um constante acréscimo no de carotenóides (Figura 5). A concentração média de clorofila diminuiu de $0,3 \mathrm{mg}$ $\mathrm{g}^{-1}$ para $0,16 \mathrm{mg} \mathrm{g}^{-1}$, enquanto a concentração média de carotenóides aumentou de $2,1 \mathrm{mg} \mathrm{g}^{-1}$ para $4,3 \mathrm{mg} \mathrm{g}^{-1}$, durante o armazenamento dos frutos. Há uma dinâmica de biogênese inversa entre os precursores cloroplastos e cromoplastos e, por conseqüência, de clorofilas e de carotenóides que são seus sucessores (Ebert \& Gross, 1985).

\section{CONCLUSÕES}

- A aplicação de $\mathrm{AG}_{3}$ e AVG possibilitou armazenar caquis 'Fuyu' em temperatura ambiente $\left(23 \pm 3^{\circ} \mathrm{C}\right.$ e $75 \pm 5 \%$ UR) por períodos de 16 e 20 dias, respectivamente. Para os frutos-controle, o armazenamento foi de 12 dias. 


\section{REFERÊNCIAS BIBLIOGRÁFICAS}

AUTIO, W.R.; BRAMLAGE, W.J. Effects of AVG on maturation, ripening, and storage of apples. Journal American Society for Horticultural Science, Alexandria, v.107, n.6, p.1074-1077, 1982.

BARROS, S.A.; RODRIGUES, J.D. Efeito de fitorreguladores na maturação da fruta de tangerinas-'Poncã' Citrus reticulata. In: CONGRESSO DE INICIAÇÃO CIENTIFICA, 3., 1994, Pelotas. Anais...p.355-356.

BEN-ARIE, R.; SAKS, Y.; SONEGO, L.; FRANK, A. Cell wall metabolism in gibberellin-treated persimmon fruits. Plant Growth Regulators, Amsterdam, n.19, p.25-33, 1996.

BEN-ARIE, R.; ZUTKHI, Y. Extending the storage life of "Fuyu" persimmon by modified-atmosphere packaging. HortScience, $\mathrm{Al}$ exandria, v.27, n.7, p.811-813, 1992.

BYERS, R.E. Peach and nectarine fruit softening following aminoethoxy-vinylglycine sprays and dips. HortScience, Alexandria, v.32, n.1, p.86-88, 1997.

DENNIS, F.G.Jr.; ARCHBOLD, D.D.; VECINO, C.O. Effects of inhibitors of ethylene synthesis or action, $\mathrm{GA}_{4+7}$, and $\mathrm{BA}$ on fruit set of apple, sour cherry, and plum. Journal American Society for Horticultural Science, Alexandria, v.108, n.4, p.570573,1983 .

EBERT, G.; GROSS, J. Carotenoid changes in the peel of ripening persimmon (Diospyros kaki) cv. Triumph. Phytochemistry, Oxford, n.24, v.1, p.29-32, 1985.

HILL, C.M.; PEARSON, J. A.; SMITH, AJ.; ROGERS, L.J. Inhibition of chlorophill synthesis in Hordeum vulgare by 3-amino 2,3dehydrobenzoic acid. Biosciences Report. n.5, p.775-781, 1985.

KADER, A. A.; ZAGORY, D.; KERBEL, E.L. Modified atmosphere packaging of fruits and vegetables. Critical Reviews in Food Science and Nutrition, Davis, v.28, n.1, p.1-33, 1989.

KANG, I.K; SUH, S.G.; GROSS, K.C.; BYUN, J.K. N-terminal amino acid sequence of persimmon fruit $\beta$-galactosidase. Plant Physiology, Rockville, n.105, p.975-979, 1994.

KENDE, H. Ethylene biosynthesis. Annual Review Plant Physiology Plant Molecular Biology, Palo Alto, v.44, p.283-307, 1993.

LOONEY, N.E. Plant bioregulators in fruit production: An overawe and outlook. Journal Korean Society Horticultural Science, Seul, v.39, n.1, p.125-128, 1998.

MITCHAM, J.E.; CRISOSTO, C.H.; KADER, A.A. Recommendations for maintaining postharvest quality. Davis: Department of Pomology, University of California, 1998. 120 p.

PECH, J.C.; LATCHÉ, A.; BALAGUÉ, C.; BOUZAYEN, M.; LELIÈVRE, J.M. Postharvest physiology of climacteric fruits: recent development in the biosynthesis and action of ethylene. Sciencia Alim., v. 14, p. 3-14, 1994.

PEREZ, A.; BEN-ARIE, R.; DINNOR, A.; GENIZI, A.; PRUSKY, $D$. Prevention of black spot disease in persimmon fruit by gibberellic acid and iprodione treatments. Phytopathology, St. Paul, v.85, n.2, p.221-225, 1995.

PETRI, J.L.; LEITE, G.B. Efeito do AVG sobre o comportamento de frutos da macieira. In: ENCONTRO NACIONAL SOBRE FRUTICULTURA DE CLIMA TEMPERADO, 2., 1999, Fraiburgo, SC. Anais... Caçador- SC, 1999.p. 57-63.

RAMOJARO, F.; BANET, E.; LORENTE, S. Carotenoids en flavedo y pulpa de pomelo. Revista Agronomica y Tecnologia de Alimentos, n.9, p.385-392, 1979. 